\title{
Effect of urea doping on spectral, optical and thermal properties of L-histidine crystals
}

\author{
C. Alosious Gonsago ${ }^{1}$, Helen Merina Albert ${ }^{2}$, R. Umamaheswari ${ }^{2}$ and A. Joseph Arul Pragasam ${ }^{2^{\star}}$ \\ ${ }^{1}$ Department of Physics, A.J. College of Engineering, Chennai-603 103, India \\ ${ }^{2}$ Department of Physics, Sathyabama University, Chennai-600 119, India \\ $2^{*}$ drjosephsu@gmail.com
}

\begin{abstract}
The organic nonlinear optical materials pure and urea-doped L-histidine crystals were grown by slow solvent evaporation method at the room temperature of $32{ }^{\circ} \mathrm{C}$. The effect of doping urea on the spectral, optical, and thermal properties of L-histidine crystals has been investigated. Single crystal X-ray diffraction study was performed to know the structural characterization of the grown crystals and it shows slight structural changes as a result of doping. The XRD data reveals that both the crystals crystallize in the non-centro symmetric space group $\mathrm{P} 2{ }_{1} 2_{1} 2_{1}$. The FT-Raman and ${ }^{13} \mathrm{C}$-NMR spectra have been recorded for qualitative analysis and identification of the compounds. The optical transmittance study was performed to analyze the transparency of the grown crystals and found that the transparency was excellent in the wavelength region $235-1200 \mathrm{~nm}$. The optical absorption coefficient $\alpha$ has been analyzed and the optical band gap of the grown crystals was estimated to be $5.28 \mathrm{eV}$. Second harmonic generation of the grown crystals of this work was confirmed by the emission of green radiation using Nd:YAG laser. The surface morphologies of the crystals were analyzed by HR-SEM analysis. Differential scanning calorimetry technique (DSC) indicates that there is no phase transition takes place below $276{ }^{\circ} \mathrm{C}$. Preliminary studies show that the grown crystals are suitable for optoelectronics applications and SHGs.
\end{abstract}

Keywords: XRD, FT-Raman, ${ }^{13} \mathrm{C}-\mathrm{NMR}$, Optical transmittance, NLO, DSC

\section{Introduction}

A continuing need persists to build up new class of nonlinear optical materials useful for frequency conversion in the UV to near IR spectral region. The nonlinear optical (NLO) materials have been widely studied, due to their significant impact in various fields like telecommunication, optical data storage, optical switching, image manipulation and processing (Robert et al., 2010, Arumanayagam \& Murugakoothan, 2011; Jothi \& Ramamurthi, 2011). High performance electro-optic switching elements of optical information processing and telecommunication are based on materials with high nonlinear optical properties. Intensive research is going on to find suitable materials for frequency conversion of IR radiation to UV wavelengths. Particularly, the materials which can generate second harmonic blue-green light using laser diodes have been attracting a great deal of interest. Organic nonlinear compounds are attracting a great deal of attention, owing to their potentially high nonlinearities, chemical flexibilities and rapid responses in electro-optic effect compared with the inorganic nonlinear compounds.

Amino acids are interesting materials for NLO applications, as they exhibit molecular chirality, absence of strongly conjugated bonds and Zwitterionic nature of the molecule. L-histidine is an optically active amino acid which behaves like a proton donor, proton acceptor and a nucleophilic reagent. The salts of $L$-histidine received a great deal of attention as promising NLO materials after the growth of L-histidine tetraflouroborate (Marcy et al., 1995). In recent years, new salts of L-histidine have been synthesized (Anandan \& Jayavel, 2011; Alosious et al. 2011; Ramajothi \& Danuskodi, 2007). It is proved that the physical properties of NLO crystals can be altered by doping with organic additives (Jayaprakash et al., 2011; Joema et al., 2011). Motivated by the earlier reports on doping with organic additives, we have grown pure and urea-doped L-histidine crystals. In this study, we report the solubility, crystal growth, single crystal XRD, FTRaman, ${ }^{13} \mathrm{C}-\mathrm{NMR}$, UV-Vis transmittance, HR-SEM, SHG and DSC studies of pure and urea-doped L-histidine crystals.

\section{Experimental}

\section{Solubility study}

Commercially available L-histidine and urea (AR grade) were used for the solubility measurements and crystal growth. The solubility study is generally carried out to know the amount of the material available for the growth and hence defines the size of the crystal that can

Fig. 1. Solubility curves of (a) pure and (b) urea-doped L-histidine samples.

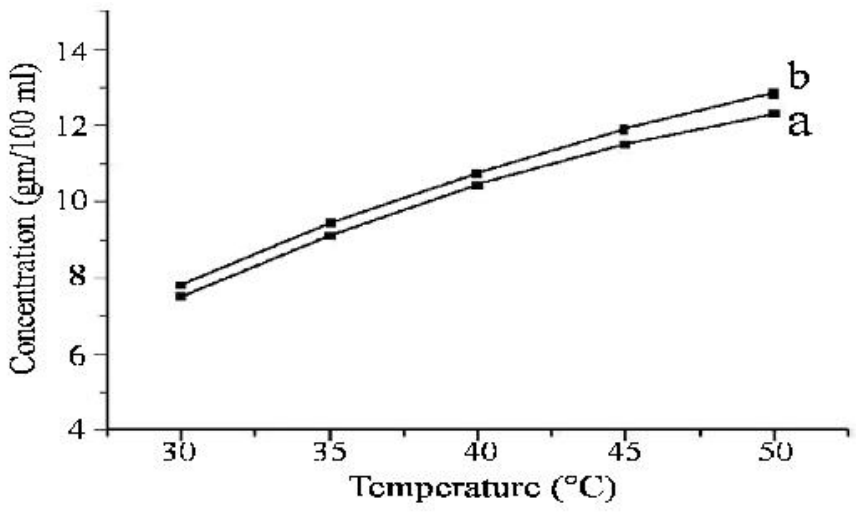

Research article

COIndian Society for Education and Environment (iSee)
"Urea-doped L-histidine crystals" http://www.indjst.org
C.A.Gonsago et al. Indian J.Sci.Technol. 
be grown (Gunasekaran \& Ponusamy, 2006). The solubility curves for pure and urea-doped L-histidine samples in water at different temperatures ranging from 30 to $50{ }^{\circ} \mathrm{C}$ are shown in Fig.1. Here, the solubility is the amount of solute in grams present in $100 \mathrm{ml}$ of saturated solution at a given temperature. From the graphs, it is observed that solubility increases with increase in temperature for both the compounds and it is found to be more for the urea-doped L-histidine. It is obvious that for the urea-doped sample, the solvent water is capable of accommodating a slightly increased amount of solute for saturation at a particular temperature. Since the solubility of the compounds increases with increase in temperature, the samples of this study have positive temperature coefficient of solubility. Hence pure and urea-doped L-histidine crystals can be grown from aqueous solution by slow solvent evaporation method.

\section{Crystal growth}

The single crystal of L-histidine with the molecular formula $\mathrm{C}_{6} \mathrm{H}_{9} \mathrm{~N}_{3} \mathrm{O}_{2}$ was grown by dissolving sufficient amount of L-histidine in deionized water at $32{ }^{\circ} \mathrm{C}$. To get homogeneous mixture, the solution was continuously stirred for about 6 hours using magnetic stirrer. The solution was then heated to dryness below an optimum temperature of $55^{\circ} \mathrm{C}$ in a temperature controllable water bath. Tiny crystalline samples were obtained after necessary heating. To obtain urea-doped sample, $2 \mathrm{wt} \%$ of urea was added to the solution of L-histidine and the same procedure was followed as that of pure L-histidine.

The tiny crystalline samples were again thoroughly dissolved in deionized water at $32{ }^{\circ} \mathrm{C}$ to form saturated solution. The solutions were filtered twice to remove unwanted impurities. The filtered solution of pure and urea-doped L-histidine were kept in two separate borosil beakers covered with porous papers. To maintain constant growth rate, the solutions were kept in a constant temperature water bath at $32{ }^{\circ} \mathrm{C}$. In this study, two drops of hydrogen peroxide were added to the solutions of pure and urea-mixed L-histidine to inhibit the growth of microorganism (Joseph et al. 2005). Crystals of adequate size and good transparency were obtained after a period of 30 days.

Results and discussion

Single crystal XRD

Table 1. Crystallographic data of pure and urea-doped Lhistidine crystals

\begin{tabular}{|c|c|c|c|}
\hline Compound & $\begin{array}{c}\text { Cell } \\
\text { parameters }(\AA)\end{array}$ & $\begin{array}{c}\text { Volume } \\
(\AA)^{3}\end{array}$ & Space group \\
\hline & $\mathrm{a}=5.176$ & & \\
\hline $\begin{array}{c}\text { Pure L- } \\
\text { histidine }\end{array}$ & $\mathrm{b}=7.320$ & 714.19 & $\mathrm{P} 2{ }_{1} 2_{1} 2_{1}$ \\
\hline & $\mathrm{c}=18.85$ & & \\
\hline & $\mathrm{a}=5.180$ & & \\
\hline & $\mathrm{b}=7.402$ & 720.45 & $\mathrm{P} 2{ }_{1} 2_{1} 2_{1}$ \\
\hline $\begin{array}{c}\text { Urea-doped } \\
\text { L-histidine }\end{array}$ & $\mathrm{c}=18.79$ & & \\
\hline & & & \\
\hline
\end{tabular}

Vol. 5 No. 3 (Mar 2012) ISSN: 0974-6846

To identify the lattice parameters and crystal structure, the grown crystals were subjected to single crystal XRD study using the instrument ENRAF NONIUS CAD4 diffractometer with the $\mathrm{MoK}_{\alpha}$ radiation source of

Fig.2. FT-Raman spectra of (a) pure and (b) ureadoped L-histidine crystals.

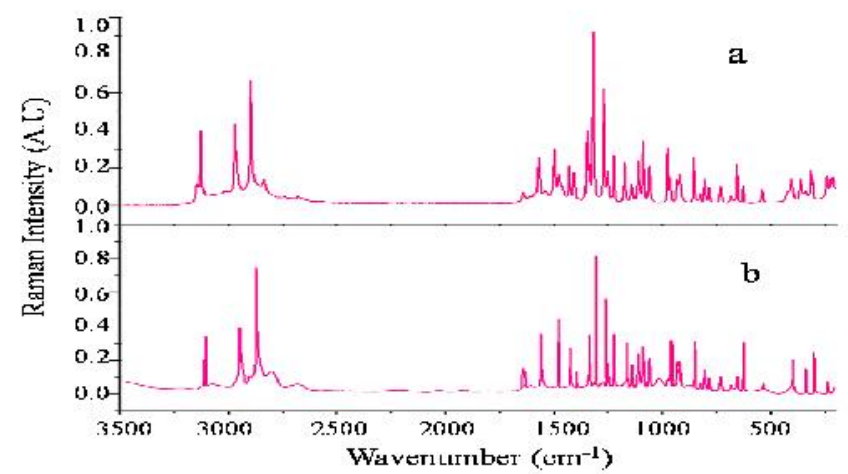

wavelength $0.7107 \AA$. It is observed that both crystals belong to orthorhombic system with the space group $P 2_{1} 2_{1} 2_{1}$. The lattice parameters for pure $L$-histidine crystal are $a=5.176 \AA, b=7.32 \AA, c=18.85 \AA, V=714.19 \AA^{3}$ and $\alpha=\beta=\gamma=90^{\circ}$. The crystallographic data of the pure Lhistidine crystal is found to be in concurrence with the reported values (Madden et al. 1972). In the case of ureadoped crystal, a slight increase in the lattice parameters and volume has been noticed. This may be due to the presence of urea in the lattice of L-histidine crystal. The crystallographic data for pure and doped L-histidine crystals are given in Table 1.

\section{FT-Raman study}

The FT-Raman spectra are very useful tool for the identification of compounds and qualitative analysis. The FT-Raman spectral analysis has been carried out at room temperature using the instrument Bruker RFS27 FTRaman spectrometer. An Nd:YAG air cooled diode pumped laser beam of power $100 \mathrm{~mW}$ was focused on to the sample. The scattered light was collected at the angle of $180^{\circ}$ in the region $200-3500 \mathrm{~cm}^{-1}$, resolution $2 \mathrm{~cm}^{-1}$, 200 scans. The recorded Raman spectra for both the crystals are shown in Fig.2. From the Fig.2a of Raman spectra, the peaks observed at 3128 and $2970 \mathrm{~cm}^{-1}$ are assigned to the $\mathrm{N}-\mathrm{H}$ and $\mathrm{C}-\mathrm{H}$ stretching vibrations of the five membered imidazole ring. But, these peaks are slightly shifted down in the case of urea-doped crystal and are appearing at 3109 and $2956 \mathrm{~cm}^{-1}$. It could be due to the lattice strain developed in the crystal as the result of doping. For the pure crystal, the $\mathrm{N}-\mathrm{H}$ bending vibration is resolved at $1429 \mathrm{~cm}^{-1}$. The asymmetric and symmetric stretching modes of the $\mathrm{CH}_{2}$ group are resolved at 2897 and $2826 \mathrm{~cm}^{-1}$. The $\mathrm{C}=\mathrm{O}$ stretching vibration is observed at $1570 \mathrm{~cm}^{-1}$. The stretching vibrations of amino group $\left(-\mathrm{NH}_{3}\right)$ are resolved at 1497 at $1429 \mathrm{~cm}^{-1}$. The C-C and $\mathrm{C}-\mathrm{O}$ stretching vibrations are observed at 1270 and 1223 $\mathrm{cm}^{-1}$. The $\mathrm{C}-\mathrm{H}$ in plane and out of plane bending vibration is observed at 1086 and $975 \mathrm{~cm}^{-1}$. The peak at $656 \mathrm{~cm}^{-1}$
Research article

CIndian Society for Education and Environment (iSee)
"Urea-doped L-histidine crystals" http://www.indjst.org
C.A.Gonsago et al. Indian J.Sci.Technol. 
is due to ring deformation. The $\mathrm{C}-\mathrm{C}$ twisting and $\mathrm{C}-\mathrm{N}$ deformations are observed at 362 and $316 \mathrm{~cm}^{-1}$. From the Fig.2b, a slight shift in some of the characteristic vibrational frequencies is observed for the doped crystal which may be due to the effect of dopant.

${ }^{13} \mathrm{C}-\mathrm{NMR}$ spectral study

Fig.3. ${ }^{73} \mathrm{C}-\mathrm{NMR}$ spectra of (a) pure and (b) urea doped L-histidine crystals
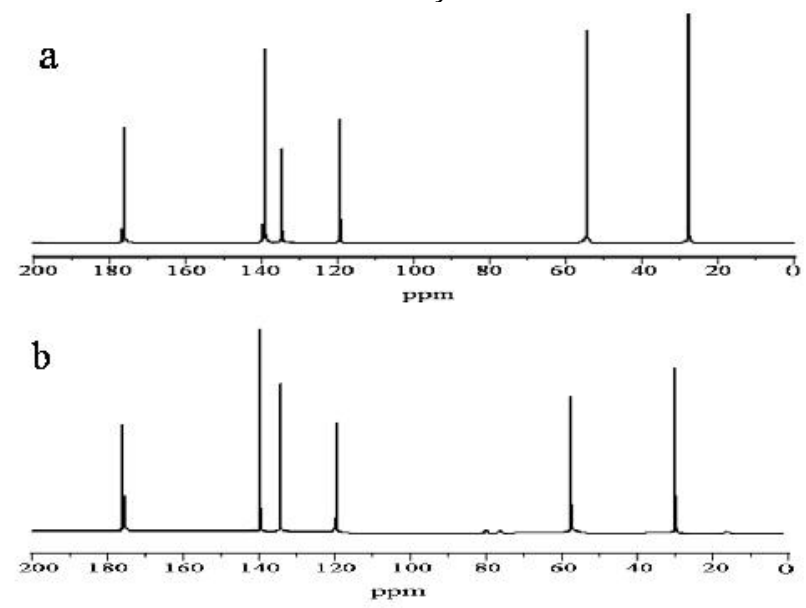

In order to ascertain the molecular structure of the grown crystal, ${ }^{13} \mathrm{C}-\mathrm{NMR}$ spectral study has been carried out using the instrument Bruker $300 \mathrm{MHz}$ (Ultrashield) TM instrument at $20^{\circ} \mathrm{C}$. The recorded spectra are shown in Fig.3. The ${ }^{13} \mathrm{C}-\mathrm{NMR}$ spectra of L-histidine show six resonance peaks. The resonance peak resolved at $\delta=$ $176.86 \mathrm{ppm}$ is due to the presence of $\mathrm{CO}$ group of side chain. Two consecutive signals resolved at $\delta=$ $139.20 \mathrm{ppm}$ and $135.21 \mathrm{ppm}$ are due to the presence of $\mathrm{CH}$ groups of imidazole ring. The peak at 119.23ppm is due the presence of $\mathrm{C}$ atom of the ring. The resonance peaks resolved at $\delta=54.43$ and $27.14 \mathrm{ppm}$ are due to the presence of $\mathrm{CH}$ and $\mathrm{CH}_{2}$ groups of the side chain of $\mathrm{L}$ histidine. In the case of urea-doped L-histidine crystals, the positions of resonance peaks were slightly shifted from pure crystal. This may be due to the presence of the dopant in the crystal. The chemical shifts in ${ }^{13} \mathrm{C}$-NMR spectra of pure and urea-doped L-histidine crystals are presented in Table 2.

Fig.3. represents the ${ }^{13} \mathrm{C}-\mathrm{NMR}$ spectra of (a) pure and (b) urea doped L-histidine crystals

Optical transmittance study

The optical transmittance study plays an important role in identifying the usefulness of an NLO material Table 2. The chemical shifts in ${ }^{13} \mathrm{C}-N M R$ spectra of pure and urea-doped $L$-histidine crystals

\begin{tabular}{|c|l|l|l|}
\hline Spectrum & $\begin{array}{c}\text { Signals at } \delta \\
\text { (ppm) for pure } \\
\text { L-histidine }\end{array}$ & $\begin{array}{l}\text { Signals at } \delta(\mathrm{ppm}) \text { for } \\
\text { Urea-doped L-histidine }\end{array}$ & Functional groups \\
\hline \multirow{3}{*}{${ }^{13}$ C-NMR } & 176.86 & 176.54 & $\mathrm{CO}$ of side chain \\
& $139.20,135.21$ & $140.15,134.86$ & $\mathrm{CH}$ of imidazole ring \\
& 119.23 & 119.54 & $\mathrm{C}$ of imidazole ring \\
& 54.43 & 57.36 & $\mathrm{CH}$ of side chain \\
\hline
\end{tabular}

Research article

CIndian Society for Education and Environment (iSee)

"Urea-doped L-histidine crystals" http://www.indjst.org
Fig.4. Optical spectra of (a) pure and (b) urea-doped Lhistidine crystals.

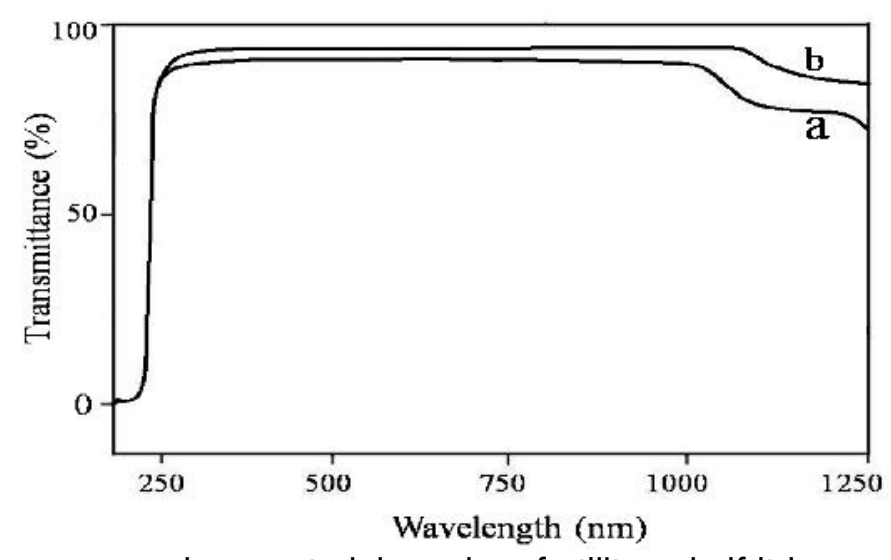

because a given material can be of utility only if it has a wide transparency window in the visible and UV regions. The UV-visible transmittance spectra of both crystals were recorded using a Varian Carry-5E UV-Vis spectrometer in the range $200-1200 \mathrm{~nm}$ and are shown in Fig.4.

From the UV-Vis spectral analysis, it is noted that the optical transmittance is very high in the range 235-1200 $\mathrm{nm}$, which is the desirable property for NLO applications (Martin et al. 2007). A sharp fall in the transmittance is observed in the spectra at around $235 \mathrm{~nm}$ for both crystals which corresponds to the lower cut-off wavelength. The lower cut-off wavelength at $235 \mathrm{~nm}$ reveals that the material is quite suitable for SHG generation and other related optoelectronic applications. From the spectra, it is observed that the lower cut-off wavelength is not altered due to doping but the percentage of transmittance is slightly enhanced in the case of urea-doped crystal.

The dependence of optical absorption coefficient $(\alpha)$ on photon energy facilitates one to study the band structure and the nature of transition of electrons. The optical band gap energy is calculated from the optical transmittance spectrum. The optical band gap is very useful aspects of the band structure, as it influences the optical and electrical characteristics of the crystal. The optical energy gap is determined from Tauc relation (Tauc, 1974; Krishnakumar et al., 2008). According to this relation, the absorption coefficient $\alpha$ for a given material is given by, $a \mathrm{hv}=\mathrm{A}\left(\mathrm{hv}-\mathrm{E}_{\mathrm{g}}\right)^{\mathrm{n}}$

where $A$ is a constant. $E_{g}$ is the band gap of the material and $\mathrm{n}$ is an index which can have the values $1 / 2,3 / 2,2$ or 3 depends upon the nature of the electronic transition. A plot is drawn between hv and (ahv) ${ }^{2}$ and is shown in Fig.5. The band gap energy of the pure and doped crystals was evaluated by exploring a straight line in the linear region of the graph at $(\alpha h v)^{2}=0($ Lucia et 
al. 2011). The estimated band gap energy of both crystals is $5.28 \mathrm{eV}$. As a result of wide band gap, the grown crystals possess wide transmittance window in the UV and visible region.

\section{Nonlinear optical study}

The SHG efficiency was measured for the grown crystals using the standard Kurtz Perry powder technique (Kurtz \& Perry, 1968). The fundamental beam of a Qswitched Nd: YAG laser with a wavelength of $1064 \mathrm{~nm}$, input energy of $3.2 \mathrm{~mJ}$, pulse duration of $8 \mathrm{~ns}$, a repetition of $10 \mathrm{~Hz}$ and a spot size of $1 \mathrm{~mm}$ diameter was used in this technique. The grown crystals were grounded in to fine microcrystalline powders and were densely packed between transparent glass slides. Microcrystalline sample of KDP was used for the comparison of conversion efficiencies of the grown crystals. The second harmonic signals generated from the crystals were confirmed by the emission of green radiation. The results obtained by this method show that the SHG efficiencies for pure and ureadoped crystals are about $25 \%$ and $29 \%$ as that of KDP.

Fig.5. Plot of (ahv)" versus photon energy hv

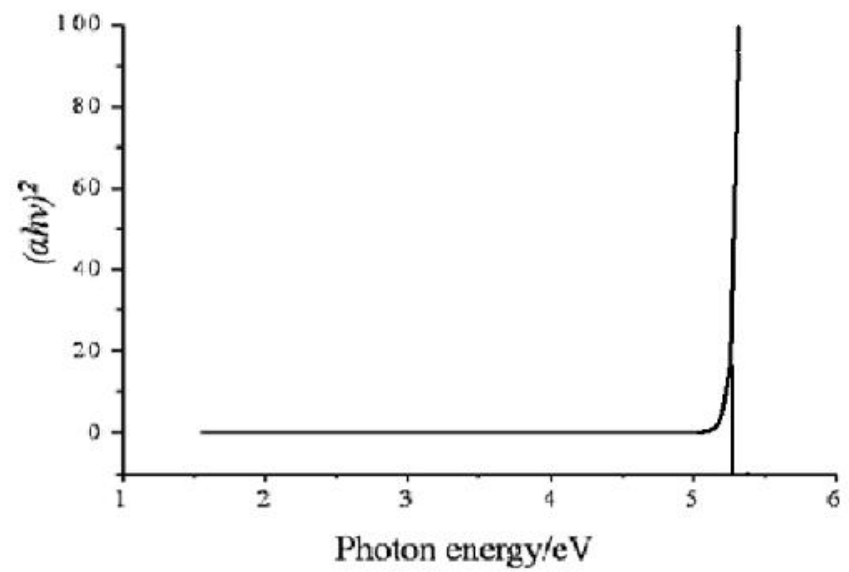

\section{HR-SEM analysis}

HR-SEM analysis has been carried out for the grown crystals to study the nature and surface morphology. The transparent regions of the crystals were cut into few $\mathrm{mm}$ for examining the surface morphology. The SEM micrographs of pure and urea-doped crystals taken in the same magnification are presented in Fig.6. The micrograph of pure L-histidine (Fig.6a) shows that the surface is smooth and continuous. The surface is free from any inclusions and it shows the purity of the compound. But the micrograph of urea-doped L-histidine (Fig.6b) shows the presence of some visible inclusions deposited on the surface of the crystal. This may be due to the presence of urea in the crystalline matrix and temperature fluctuations during the growth process.

\section{Thermal analysis}

Differential scanning calorimetry (DSC) technique was used to study the water of crystallization, phase transition, and thermal decomposition of the compound (Prakash et al. 2008).The DSC analysis was carried out in the temperature range $20-300{ }^{\circ} \mathrm{C}$ at a heating rate of
Fig.6. HR-SEM micrographs of (a) pure and (b) ureadoped L-histidine crystals.
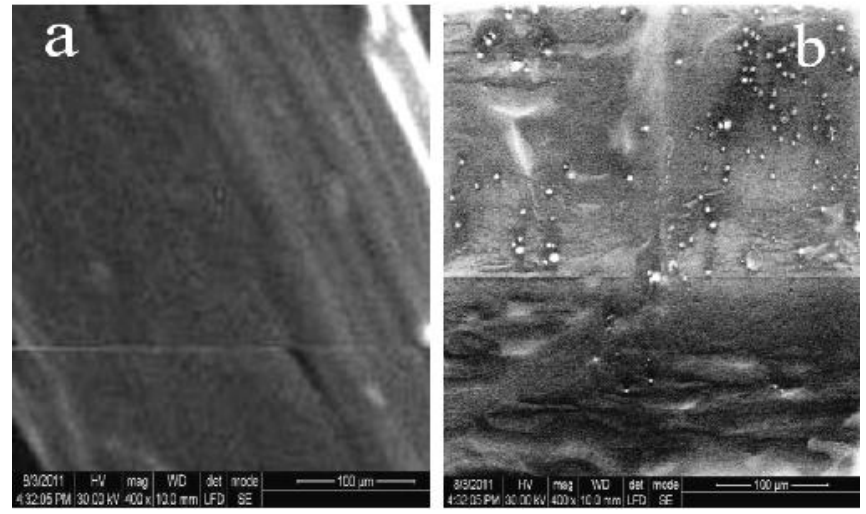

$20{ }^{\circ} \mathrm{C} / \mathrm{min}$ under the nitrogen atmosphere using the instrument NETZSCH DSC 204. The typical DSC curves of both crystals are shown in Fig.7. The DSC trace is smooth up to $260{ }^{\circ} \mathrm{C}$ and then shows a sharp endothermic peak. For pure crystal, the endothermic peak is resolved at $276{ }^{\circ} \mathrm{C}$ which represents the melting point. In the case of urea-doped crystal, the endothermic peak is resolved at $279{ }^{\circ} \mathrm{C}$ which represents the melting point of the doped crystal. Another notable observation is that there is no exothermic or endothermic peaks are found below this temperature and hence the crystal is completely free from water of crystallization. The DSC study clearly shows that the grown crystals are thermally more stable and the melting point is slightly increased in the case of doped crystal.

Conclusions

In this study, pure and urea-doped L-histidine single crystals were grown by slow evaporation method at the room temperature of $32{ }^{\circ} \mathrm{C}$ in the period of 30 days. The single crystal XRD study indicates that the grown crystals belong to orthorhombic system with the space group $\mathrm{P} 2{ }_{1} 2_{1} 2_{1}$. The crystallographic data shows a slight increase in the lattice parameters and volume for the

Fig. 7. DSC curves of (a) pure and (b) urea-doped Lhistidine crystals.

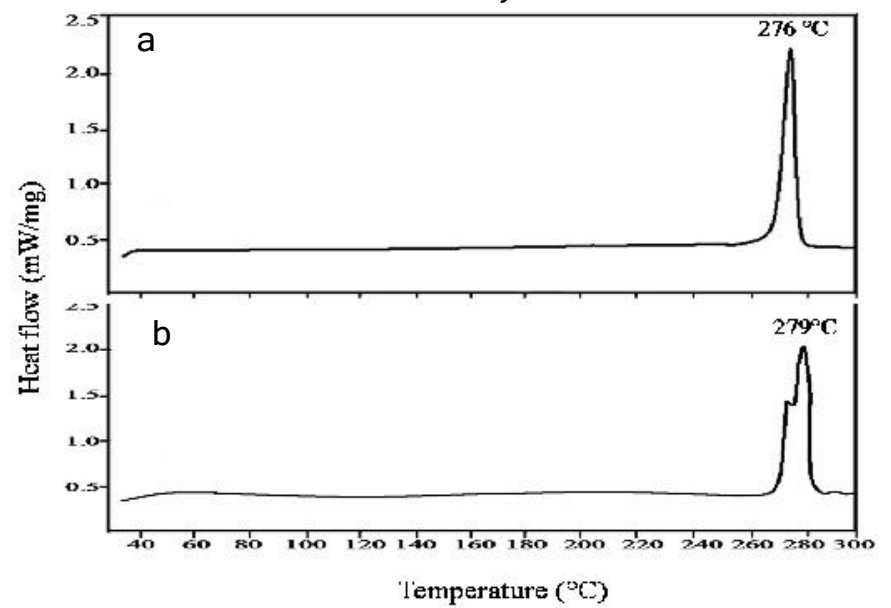


doped crystal. The molecular structures of the grown crystals were confirmed by FT-Raman and ${ }^{13} \mathrm{C}-\mathrm{NMR}$ spectral analysis. The optical transmission study reveals that the grown crystals are highly transparent in the region 235-1200 $\mathrm{nm}$ and the percentage of transmittance is slightly enhanced in the case of urea-doped crystal. Good transmittance observed in the UV and visible region enables the crystals suitable for optoelectronics applications and SHGs. The optical band gap energies for both crystals are found to be almost same and the calculated value is $5.28 \mathrm{eV}$. The SHG test confirms the NLO property of the crystals. The NLO efficiency is marginally increased in the urea-doped crystal. The DSC study reveals that both crystals are highly stable and the melting point of the urea-doped crystal is slightly increased as a result of urea dopant.

\section{References}

1. Alosious Gonsago C, Helen Merina Albert, Malliga P and Joseph Arul Pragasam A (2012) Crystallization, spectral, and thermal characterization of L-histidine methyl ester dihydrochloride (LHMED). J. Therm. Anal. Calorim. 107, 1231-1235.

2. Arumanayagam $T$ and Murugakoothan $P$ (2011) Studies on optical, mechanical properties of new organic NLO crystal: Guanidinium 4-aminobenzoate (GuAB) Mater. Lett. 65, 2748-2750.

3. Gunasekaran S and Ponusamy MN (2006) Growth and characterization of cadmium magnesium tetra thiocyanate crystals. Cryst. Res. Technol. 41 (2), 132137.

4. Jayaprakash Manoharan AJ, Joseph John N, Revathi V, Rajendran KV and Andavan PM (2011) Effect of amino acid doping on the dielectric properties of triglycine sulphate (TGS) crystals. Indian J. Sci. Technol. 4 (6), 688-691.

5. Joema SE, Perumal S, Ramalingam $S$, Selvarajan $P$ (2011) Studies on structural, optical, mechanical and thermal properties of undoped and urea doped Lhistidine bromide (LHB) single crystals. Recent Res. Sci. Technol. 3(3) 63-68.

6. Joseph Arul Pragasam A, Selvakumar S, Thamizharasan K, Prem Anand D and Sagayaraj $P$ (2005) Growth and optical characterization of $\mathrm{Cu}^{-}$and $\mathrm{Mg}^{-}$substituted L-arginine di phosphate single crystals. J. Cryst.Growth. 280, 271-278.

7. Jothi L and Ramamurthi K (2011) Growth and characterization of an organic NLO crystal:4-chloro4-methyl benzylidene aniline. Indian J. Sci. Technol. 4 (6), 666-669.

8. Krishnakumar V, Sivakumar S, Nagalakshmi R, Bhuvaneswari S and Rajaboopathi M (2008) Effect of doping an organic molecule ligand on TGS single crystals. Spectrochim. Acta A. 71, 480-485.

9. Kurtz SK and Perry TT. (1968). A powder technique for the evaluation of non-linear optical materials. $J$. Appl. Phys. 39, 3798-3813.
Vol. 5 No. 3 (Mar 2012)

ISSN: 0974- 6846

10. Lucia Rose ASJ, Selvarajan P and Perumal S (2011) Growth, structural, mechanical, thermal and dielectric characterization of phosphoric acid admixed Lalanine (PLA) single crystals. Spectrochim. Acta A. $81,270-275$.

11. Madden JJ Edward L, Mcgandy and Nadrian, Seeman C (1972) The crystal Structure of the orthorhombic form of L-(+)-Histidine. Acta Crystallogr. 28, 2377-2380.

12. Marcy HO, Rosker MJ, Warren LJ, Cunningham PH and Thomas CA (1995) L-histidine tetrafluoroborate: a solution-grown semiorganic crystal for nonlinear frequency conversion, Opt. Lett., 20 (3), 252-254.

13. Martin Britto Dhas SA, Suresh M, Bhagavannarayana $G$ and Natarajan S (2007) Growth and characterization of L- Tartaric acid, an NLO material. J. Cryst. Growth. 309, 48-52.

14. Pandurangan Anandan and Ramasamy Jayavel (2011) Crystal growth and characterization of semiorganic single crystals of L-histidine family for NLO applications. J. Cryst. Growth. 322, 69-73.

15. Ramajothi J and Danuskodi S (2007) Crystal growth, thermal and optical studies on a semiorganic nonlinear optical material for blue-green laser generation. Spectrochim. Acta A. 68, 1213-1219.

16. Robert R, Justin Raj C, Krishnan S, Uthrakumar R, Dinakaran S and Jerome Das S. (2010). Spectral,optical and mechanical studies on Lhistidine hydrochloride monohydrate (LHC) single crystals grown by unidirectional growth technique. Physica B. 405, 3248-3252.

17. Tauc J (1974) Amorphous and liquid semiconductors, Plenum press, NY.

18. Thomas Joseph Prakash J Vijayan $\mathrm{N}$ and Kumararaman S (2008) Growth and characterization studies on glycine barium dihydrochloride single crystals for NLO applications. Spectrochim. Acta A. $71,1250-1252$.
"Urea-doped L-histidine crystals" http://www.indjst.org
C.A.Gonsago et al. Indian J.Sci.Technol. 